\title{
Anemia nas Doenças Inflamatórias Intestinais
}

\author{
Antonio Carlos Moraes, Cyrla Zaltman, \\ Flavia de Alvarenga Netto, Clarisse Lobo
}

A anemia é uma manifestação extra-intestinal frequente que contribui para presença de sintomas pouco específicos e por vezes debilitantes, como a fadiga crônica, com impacto negativo na qualidade de vida com a mesma intensidade que a diarréia e/ou a dor abdominal. Entretanto, esta condição tende a ser sub-diagnosticada assim como inadequadamente tratada por gastroenterologistas e cirurgiões, apesar da eficácia das opções terapêuticas existentes. ${ }^{1}$ A prevalência desta condição é bastante variável, dependendo da definição empregada e do tipo de população estudada (ambulatorial versus hospitalizada) ${ }^{2}$.

Recente metanálise envolvendo 2192 pacientes europeus portadores de DII, demonstrou uma prevalência global de anemia de 24\%. O uso de medicação especifica e a presença de atividade de doença foram considerados fatores associados à presença de anemia, sendo mais frequente em pacientes com $\mathrm{DC}$ do que com $\mathrm{RCU}^{3}$. Entretanto, pacientes em fase de remissão também podem apresentar anemia, deficiência de ferro, de vitamina B12 e/ou ac fólico ${ }^{4}$. A anemia existente na DII decorre principalmente da deficiência de ferro, que pode ser secundária a restrições dietéticas, disabsorção, a perda entérica e a presença da inflamação intestinal. Outros fatores menos frequentes também podem estar implicados como o uso de medicações especificas empregadas no tratamento da DII 
(sulfassalazina, Metrothexato, Tiopurinas) e a presença de anemia hemolítica auto-imune mais freqüente em pacientes com RCU ${ }^{5}$.

Devido a complexidade do tema, a identificação da deficiência de micronutrientes e da anemia assim como os possíveis mecanismos envolvidos, se torna necessária e favorece a introdução precoce da terapêutica adequada. A atuação de uma equipe multidisciplinar visando a abordagem integrada na correção destas deficiências assim como o controle da atividade de doença é fundamental.

\section{Deficiência de Ferro (DF)}

\section{Conceitos e causas}

A causa mais comum de anemia, em todo o mundo, é a eritropoiese ferro-restrita, consequente a um ou mais tipos de deficiência de ferro (DF). Recentemente foi proposta a diferenciação entre: deficiência absoluta de ferro (DAF), deficiência funcional de ferro (DFF) e anemia de doença crônica (ADC) ou sequestro de ferro ${ }^{6}$. Na DII, a anemia habitualmente é a expressão da associação de deficiência de ferro e anemia de doença crônica, que serão descritas a seguir ${ }^{7}$.

Deficiência Absoluta de Ferro (DAF) considerada como déficit no teor total de ferro do organismo. Ocorre quando as exigências de ferro excedem o seu fornecimento ou quando as perdas são maiores do que a ingestão dietética. Podem ser consideradas possíveis causas: 1 ) Ingestão inadequada na dieta (alcoolismo crônico, desnutrição proteico-calórica); 2) Demanda aumentada de ferro (gravidez, menstruação, hemorragia digestiva); 3) Absorção gastrointestinal inadequada (síndrome de má-absorção, uso de medicamentos) $)^{8.9}$.

Deficiência Funcional de Ferro (DFF) surge quando a mobilização de ferro não é rápida o suficiente para atender a maior demanda durante estimulação acentuada pela eritropoietina endógena ou medicamentosa (EPO). A taxa de liberação do ferro proveniente das células do sistema reticulo endotelial é limitada, e esta pode não conseguir atender as necessidades dos glóbulos vermelhos, por este motivo, esta deficiência pode ocorrer mesmo com o suprimento de ferro adequado para eritropoiese basal ${ }^{10}$. 
Anemia de Doença Crônica (ADC) ou Sequestro de Ferro corresponde à redução na disponibilização de ferro para o plasma, por bloqueio na liberação do ferro dos macrófagos do sistema reticulo-endotelial (SRE), dos enterócitos (absorção dietética de ferro) e/ou dos hepatócitos (armazenamento de ferro).

Goodnough e col$^{6}$ descrevem a desregulação da homeostasia do ferro existente nas enfermidades inflamatórias como "Síndrome de Sequestro de Ferro", que seria secundária a ativação imunológica aguda ou crônica. Esta síndrome também pode estar presente em processos infecciosos agudos ou crônicos, malignidades e alterações auto-imunes. Durante a fase aguda da DII, a hepcidina, uma proteína de fase aguda hepática é induzida por citocinas, como o TGF-ß e IL-6, assim como pela camada lipopolissacaridea de bactérias. Esta proteína controle da absorção de ferro no duodeno. Quando aumentada pode acarretar a redução da absorção assim como o bloqueio a liberação deste micronutriente dos macrófagos ${ }^{11}$.

\section{Metabolismo e homeostase do ferro}

O metabolismo do ferro envolve várias etapas importantes, incluindo a regulação e absorção intestinal de ferro, o transporte de ferro para as células, o armazenamento de ferro, a incorporação do ferro às proteínas e a reciclagem do ferro após a degradação dos glóbulos vermelhos. Em condições normais, a ausência de mecanismo ativo de excreção de ferro, torna a homeostase do ferro estritamente controlada pela absorção intestinal.

O ferro iônico existe sob a forma de ferro ferroso reduzido $\left(\mathrm{Fe}^{+2}\right)$ ou ferro férrico oxidado $\left(\mathrm{Fe}^{+3}\right)$. Uma dieta balanceada contem de 12 a $18 \mathrm{mg}$ ferro/dia , se apresentando sob a forma heme e não-heme, mas apenas 1 a 2 mg deste ferro ingerido é efetivamente absorvido. 0 ferro heme está presente em alimentos de origem animal (carne bovina, frango e peixe), e o ferro não-heme, além de ser ofertado pela carne vermelha, também é encontrado nos cereais e vegetais. O ferro heme é solúvel nas condições habituais do intestino delgado, sendo facilmente absorvido pela mucosa intestinal. A biodisponibilidade desta forma de ferro da dieta é geralmente elevada e não é afetada pelos inibidores de absorção de ferro na dieta, tais como polifenóis e de fitato ou agentes quimicos ${ }^{12}$. 
O conteúdo médio de ferro no organismo varia entre 3-4 g e é distribuído entre os glóbulos vermelhos, sistema retículo endotelial (SRE), medula óssea, músculo e fígado, existindo um equilíbrio da quantidade de ferro entre os diversos compartimentos. 0 ferro, que normalmente é absorvido no duodeno e jejuno superior, sofre a influência da ação combinada da proteína hepcidina e da citocina TNF alfa. A hepcidina, proteína que reflete o nível de ferro armazenado no organismo tem sua produção regulada pela presença de inflamação e pelo ferro existente no organismo. Na DII, a hepcidina tende a estar aumentada e portanto favorecendo uma redução na absorção de ferro intestinal (via ferroportina). Esta absorção ocorre sob a sua forma ferrosa, mais facilmente absorvivel que outra forma de apresentação existente que é a férrica, insolúvel cuja absorção se torna mais dificultada.

O ferro é liberado quando em contato com o ph ácido do estomago e reduzido a forma ferrosa que será absorvida. Este ferro é oxidado e transformado em estado férrico, que se liga a apo-ferritina. 0 ferro, ligado a ferritina, é perdido pela luz intestinal a cada 5 dias, através da descamação das células intestinais, sendo esta de pequena monta, cerca de 1 a $2 \mathrm{mg}$ de ferro / d considerando também as perdas decorrentes do fluxo menstrual e da esfoliação de células da pele ${ }^{13}$.

Cerca de 3-4 mg do ferro orgânico se encontra ligado à transferrina sérica, que transporta o ferro do intestino delgado para os locais onde será reutilizado, especialmente a medula óssea onde ocorre a formação de novos eritrócitos.

\section{Diagnóstico}

\section{a- Diagnóstico clinico}

Os pacientes com DII recém diagnosticados apresentam maior risco de desenvolvimento de anemia, por apresentarem maior atividade de doença e possivelmente sangramento assim como maior extensão de doença, no caso de RCU ${ }^{14}$.

Os níveis baixos de ferro reduzem a produção de energia do organismo, uma vez que é o componente funcional presente não apenas da hemoglobina $(\mathrm{Hb})$, mas também em grande número de enzimas importantes para as principais vias metabólicas. Os sintomas comuns 
são fadiga, esgotamento, irritabilidade e redução do desempenho físico, redução do desempenho mental e cognitivo, falta de concentração assim como a maior susceptibilidade a infecções ${ }^{11,15,16}$.

\section{b- Diagnóstico laboratorial}

O rastreamento básico de anemia inclui anemia na DII deve conter hemoglobina (hb), hemograma completo (incluindo VCM, HCM e plaquetas), dosagem de reticulócitos, ferro sérico, ferritina, saturação de transferrina e o nível de inflamação (PCR) Entretanto outros exames devem ser solicitados visando o diagnóstico diferencial como LDH, acido fólico, vitamina B12, creatinina sérica, haptoglobina e Coombs (na suspeita de hemólise) ${ }^{17}$.

\section{Deficiência Absoluta de Ferro (DAF)}

É reconhecida laboratorialmente pelos baixos níveis de ferritina sérica (<30-100 ng/mL) e baixa Saturação de Transferrina ST (<20\%). Importante lembrar que a hemoglobina sérica normal não exclui a deficiência de ferro, pois para que esta se modifique precisa ter uma perda considerável de ferro antes de haver modificações na concentração de hemoglobina. Nesta fase o individuo já pode referir fadiga, considerado sintoma pouco especifico ${ }^{18}$.

Embora a evolução da DAF para anemia por deficiência de ferro (ADF) seja um processo contínuo, três estágios de depleção de ferro podem ser descritos:

- Estágio I: Deficiência de Ferro - Ferritina < 30 g/Í sem anemia.

- Estágio II: Eritropoiese diminuída por deficiência de ferro - ferro sérico<60 $\mu \mathrm{g} /$ Í; protoporfirina das hemácias > $70 \mu \mathrm{g} /$ dí e do receptor de transferrina sérica (sTfR > $7 \mathrm{mg்/I}$ ); a saturação de transferrina (ST) < 20\% ; a capacidade total de ligação de ferro (CTLF ou TIBC) está elevada, enquanto os níveis de Hb permanecem dentro do normal.

- Estágio III: Anemia por Deficiência de Ferro - nesta fase, os níveis de ferro no organismo encontram-se tão reduzidos que os níveis de hb diminuem ( $\mathrm{hb}<12 \mathrm{~g} / \mathrm{dl}$ em mulheres e hb <13 g்/dí em homens). Neste estágio, considerado avançado de ADF, as hemácias apresentam volume corpuscular médio (VCM) < 80 (microcitose) e concentração de hemoglobina corpuscular média $(\mathrm{CHCM})<28$ pg (hipocromia) e aumento de reticulócitos hipocrômicos. Além 
desses, são achados laboratoriais peculiares dessa fase, os níveis muito baixos de ferro e de Saturação de Transferrina (ST) ${ }^{1}$.

\section{Deficiência Funcional de Ferro (DFF)}

As características da DFF são descritas na Tabela 1.

\section{Tabela 1: Características da Deficiência Funcional de}

\begin{tabular}{|l|l|}
\hline \multirow{4}{*}{$\begin{array}{l}\text { Resposta inadequada da } \\
\text { hemoglobina ao AEE* }\end{array}$} & Ferritina sérica $>30 \mu \dot{g} / l$ \\
\hline & Saturação da transferrina <20\% \\
\hline & Hemácias hipocrômicas \\
\hline & Reticulocitose \\
\hline VCM†, ou CHCM ‡ reduzidos, em casos graves \\
\hline & Resposta à suplementação de ferro EV \\
\hline
\end{tabular}

*AEE: agentes estimulantes da eritropoiese; ${ }^{\dagger}$ VCM: volume corpuscular médio; ${ }^{\ddagger} \mathrm{CHCM}$ : concentração de hemoglobina corpuscular média. Adaptado de Crichton e col. ${ }^{19}$

\section{Sequestro de Ferro - Anemia de Doença Crônica (ADC)}

Geralmente caracterizada por anemia normocromica e normocitica leve a moderada, com ferritina normal ou elevada. Em processos inflamatórios, os níveis de ferritina sérico não expressam exatamente o ferro armazenado, por sofrer a influência das citocinas inflamatórias, A concentração de ferro sérico e a saturação de transferrina estão reduzidas. Outros parâmetros utilizados para a distinção entre as alterações do ferro podem ser melhor visualizadas na tabela 2.

Tabela 2: Parâmetros para a distinção entre as alterações do ferro:

\begin{tabular}{|l|c|c|c|c|}
\hline \multicolumn{1}{|c|}{ Alterações de Ferro } & Hb & VCM & Ferritina & ST \\
\hline Deficiência de Ferro (DF) & N & N & $\downarrow$ & N \\
\hline Eritropoiese Deficiente de Ferro (EDF) & N & N & $\downarrow$ & $\downarrow$ \\
\hline Anemia por Deficiência de Ferro & $\downarrow$ & N ou $\downarrow$ & $\downarrow$ & $\downarrow$ \\
\hline Deficiência Funcional de Ferro1,3 (DFF) & $\downarrow$ & N ou $\downarrow$ & N ou & $\downarrow$ \\
\hline Anemia de Doença Crônica (ADC) & $\downarrow$ & N ou $\downarrow$ & N ou $\downarrow$ & $\downarrow$ \\
\hline ADC + DF & $\downarrow$ & N ou $\downarrow$ & $\mathrm{N}$ ou $\downarrow$ & $\downarrow$ \\
\hline
\end{tabular}

N: normal; $\uparrow$ : aumentada; $\downarrow$ : diminuída; VCM: volume corpuscular médio; ST: saturação da transferrina; Adaptado de Goodnough e col ${ }^{6 .}$

\section{Tratamento da deficiência de ferro / Anemia por deficiência de ferro}

O ponto crucial do tratamento é o controle da doença de base, fonte desencadeadora da anemia, seja por deficiência de ferro, seja por do- 
ença crônica. No entanto, quando a anemia é grave, sem tempo para se obter o controle da doença de base e o tratamento da anemia deve ser instituído. É evidente que a reposição de ferro deve ser efetuada, quando a concentração de hemoglobina estiver abaixo do normal.

A transfusão de sangue dever ser utilizada nos casos em que os parâmetros clínicos e laboratoriais mostrem risco de vida para o paciente. No entanto, a transfusão não corrige a causa da anemia e não apresenta longa sustentabilidade. São particularmente úteis se $\mathrm{hb}<8 \mathrm{~g} / \mathrm{dl}$ ou anemia com risco de vida ( $\mathrm{hb}<6,5 \mathrm{~g} / \mathrm{dl}$ ) devido a ADC agravada por sangramento ou ADF. Entretanto, a maioria dos pacientes com DII apresentam anemia leve a moderada (hemoglobina $>10 \mathrm{~g} / \mathrm{dl}$ ), mas com a perpetuação do sangramento, esses níveis podem baixar rapidamente.

Com o intuito de se promover o tratamento adequado da anemia, deve-se distinguir entre anemia por deficiência de ferro e deficiência de ferro da anemia por doença crônica. A anemia por doença crônica sem deficiência de ferro é muito rara e só é encontrada depois de reposição significativa de ferro venoso.

\section{Suplementação dietética de ferro}

Os pacientes com DII que são submetidos a restrições alimentares, geralmente consomem uma quantidade inadequada de ferro, visto que os alimentos ricos em ferro aumentam o desconforto abdominal e a distensão gasosa, dificultando o consumo ${ }^{4}$.

A concentração de ferro na dieta balanceada é usualmente de 5 a $7 \mathrm{mg} / 1000$ calorias, correspondendo a uma ingestão diária total de ferro entre 12-18 mg, Entretanto, dietas da população européia podem ter concentração de até $20 \mathrm{mg} /$ dia de ferro, mas com controvérsias devido a possibilidade de haver uma sobrecarga de ferro. ${ }^{20}$

As principais fontes de ferro alimentar são a carne vermelha, leguminosas, vegetais folhosos verdes, miúdos, frutas secas e gema de ovo. O ferro heme está presente em alimentos de origem animal (carne bovina, frango e peixe) enquanto que o não heme, principalmente nos vegetais, cereais, leguminosas e alimentos fortificados (cereais e farinhas). Em indivíduos normais, cerca de 15\% do ferro heme ingerido é absorvido, entretanto em indivíduos com deficiência de ferro, a taxa de absorção pode chegar a 35 \%. Em contraste, a absorção do ferro não-heme é bem menor, de cerca de 1 a $5 \%$. Esta forma pode ter sua ab- 
sorção influenciada pela presença de fatores de inibição ou reforço. Os fitatos (farelos, sementes), oxalatos (frutas, vegetais), polifenóis (chás, vinhos, alguns cereais), cálcio oral, e medicações como bloqueadores de bomba de prótons, inibem a absorção do ferro não heme. Carnes e vitamina $\mathrm{C}$ exercem um efeito de reforço. Importante ressaltar que a biodisponibilidade de ferro pode variar respectivamente de $15 \%$ a $5 \%$ para dietas ricas ou pobres em vitamina C e proteínas animais ${ }^{21,22,23}$.

\section{Tratamento com ferro oral}

A suplementação de ferro deve ser considerada em todos os pacientes com DII e DF e que evoluam com baixas concentrações de hemoglobina. Devido ao seu baixo custo e método não invasivo de administração de ferro por via oral em uma dose diária compreendida entre 50 mg e 200 mg tende a ser uma abordagem convencional. A quantidade máxima de ferro oral capaz de ser absorvido pelo intestino é de $20 \mathrm{mg} /$ dia. Portanto, doses maiores de ferro oral não são efetivamente absorvidos e o seu excesso pode ser tóxico para a mucosa intestinal podendo ativar a doença inflamatória.

O ferro oral é uma forma conveniente de tratamento. Entretanto, há várias limitações nessa modalidade de reposição como, absorção limitada, baixa velocidade de fornecimento de ferro e tempo prolongado de reposição do estoque. 0 estresse oxidativo das preparações baseadas em sais ferrosos é o principal fator apontado para a intolerância Gl que ocorre em 10\% a 40\% dos pacientes (dependendo do tipo de preparação) e a subsequente redução da adesão ao tratamento. Além disso, a suplementação de ferro oral não pode atender às crescentes demandas de ferro nos esquemas terapêuticos com associação a agentes estimulantes da eritropoiese.

Entretanto diretrizes demonstram que a via preferida de suplementação de ferro em pacientes com DII é a venosa, a qual mostrou ser rapidamente eficaz, de maior tolerância com impacto positivo na meIhora da qualidade de vida se comparada à reposição por via oral ${ }^{24}$.

O tempo ótimo de suplementação de ferro nas anemias relacionadas a DII não está bem estabelecido. Habitualmente demora de 2 a 3 meses para níveis de hemoglobina se normalizarem, mas para a reposição dos estoques de ferro podem ser necessários até 6 meses. Advoga-se a continuação do tratamento por 3 meses se a doença es- 
tiver controlada, caso contrário utilizar por longo prazo a dose mínima eficaz e de maior tolerância (por exemplo, sulfato ferroso $200 \mathrm{mg} / \mathrm{d}$ ) que, em idosos pode ser de até 20 a 50mg/d²5.

\section{Tratamento com ferro endovenoso}

$O$ tratamento com ferro endovenoso (EV) é mais adequado para aqueles pacientes incapazes de tolerar as quantidades necessárias de ferro oral ou quando, a perda crônica de ferro excede a velocidade de reposição possível com suplementação de ferro oral. O ferro endovenoso recomendado caso o tratamento com ferro oral não seja eficaz. Ele deve ser aplicado sempre que um efeito rápido for necessário, ou para evitar transfusão de sangue. Doses menores de EPO podem ser utilizadas quando usadas em associação com o ferro venoso, reduzindo deste modo os custos do tratamento.

Níveis baixos de transferrina ou eritropoietina são bons preditores da necessidade de agentes estimulantes da eritropoiese. A maioria dos estudos é feita com sacarato de ferro, mas a nova formulação de ferro carboximaltose tem se mostrado bastante eficaz em diversos estudos clínicos ${ }^{26}$. Algoritmos para reposição de ferro podem ser visualizados na figura 1.

\section{Figura 1: Conduta na reposição do Ferro nas}

\section{Doenças Inflamatórias Intestinais}

\section{ANEMIA POR DEFICIÊNCIA DE FERRO LEVE A MODERADA}

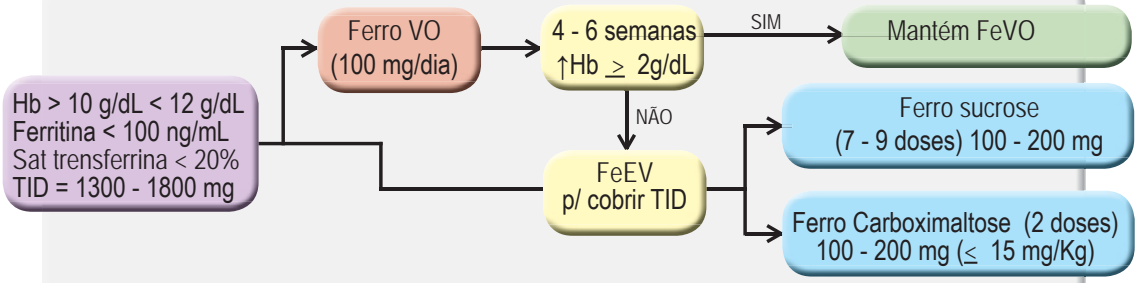

ANEMIA POR DEFICIÊNCIA DE FERRO GRAVE

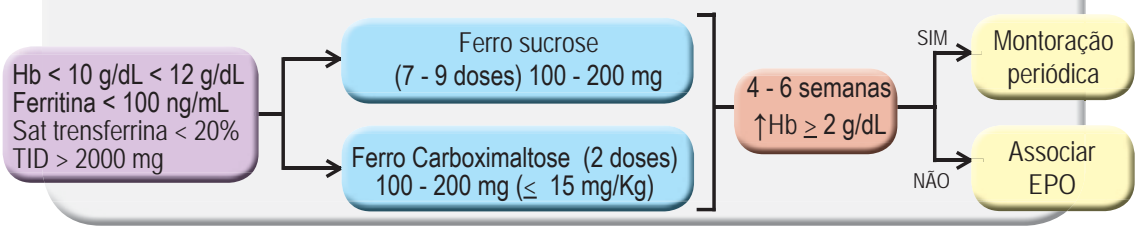

(*) $T I D=$ Total Iron Deficit $H b=$ hemoglobina; Sat Transferrina = Saruração da transferrina; $F e=$ ferro; $T I D=$ Deficit Total de Ferro (Total Iron Deficit). Retirado do algoritmo original o sal isomaltose de ferro, que não é disponível no Brasil. Adaptado de Reinisch W e col$^{27}$ 
A tabela 3 mostra as principais características dos sais de ferro endovenosos disponíveis no Brasil

\section{Tabela 3: Características dos sais endovenosos de ferro disponíveis no Brasil.}

\begin{tabular}{|c|c|c|}
\hline & Ferro Carboximaltose & Ferro Sacarose \\
\hline Diluição & $\begin{array}{l}\text { Diluir em solução } \mathrm{NaCl} 0,9 \% \\
100-200 \mathrm{mg}=50 \mathrm{ml} \\
200-500 \mathrm{mg}=100 \mathrm{ml} \\
500-1.000 \mathrm{mg}=250 \mathrm{ml}\end{array}$ & $\begin{array}{l}\text { Diluir cada ampola ( } 5 \mathrm{ml}=100 \mathrm{mg}) \\
\text { em, no mínimo } 1000 \mathrm{ml} \text { de salina }\end{array}$ \\
\hline $\begin{array}{l}\text { Tempo de } \\
\text { administração }\end{array}$ & $\begin{array}{l}\text { Correr cada } 500 \mathrm{mg} \text { em } 5 \mathrm{~min} \text {. Sem } \\
\text { necessidade de pré-teste }\end{array}$ & $\begin{array}{l}\text { Cada } 5 \mathrm{ml} \mathrm{em} 15 \mathrm{~min} . \\
\text { Sem necessidade de pré-teste }\end{array}$ \\
\hline $\begin{array}{l}\text { Dose } 35-70 \\
\text { Kg de peso }\end{array}$ & $\begin{array}{l}\mathrm{Hb}>10=1.000 \mathrm{mg} \\
\mathrm{Hb}<10=2.000 \mathrm{mg}\end{array}$ & \multirow{2}{*}{$\begin{array}{c}\text { (Hb desejada }-\mathrm{Hb} \text { atual }) \times 200+500 \\
100 \\
\text { Exemplo: }(12-10) \times 200+500 / 100 \\
=09 \text { ampolas }\end{array}$} \\
\hline $\begin{array}{l}\text { Dose }>70 \mathrm{Kg} \\
\text { de peso }\end{array}$ & $\begin{array}{l}\mathrm{Hb}>10=1.500 \mathrm{mg} \\
\mathrm{Hb}<10=2.000 \mathrm{mg}\end{array}$ & \\
\hline $\begin{array}{l}\text { Observações } \\
\text { Específicas }\end{array}$ & $\begin{array}{l}\text { Dose Máxima por dose / semana = } \\
1.000 \mathrm{mg} \text { ou Máximo } 20 \mathrm{mg} / \mathrm{Kg} \text { de peso }\end{array}$ & $\begin{array}{l}\text { Dose máxima por dose }=200 \mathrm{mg} \\
\text { Dose Máxima por semana }-500 \mathrm{mg}\end{array}$ \\
\hline
\end{tabular}

$\mathrm{Hb}=$ hemoglobina; $\mathrm{NaCl}=$ solução de Cloreto de Sódio

Nos pacientes que não obtiveram resposta adequada com doses elevadas de ferro intravenoso, pode-se prescrever eritropoetina humana recombinante ${ }^{28}$.

Bergamaschi e colaboradores ${ }^{29}$ verificaram que 2/3 de uma amostra de 263 pacientes com DII apresentavam anemia sendo a maioria portadores de doença de Crohn. O tratamento adequado para a DII aumentou progressivamente o nível de hemoglobina, mas 35\% dos pacientes permaneceram com anemia, demonstrando ser uma alteração frequente e, na maioria das vezes subestimada no tratamento destes pacientes. De forma interessante e previsível, estes mesmos autores demonstraram a melhora da anemia no período de 2 semanas após início da infusão com Infliximabe nos pacientes com DC. Em paralelo foi verificada a melhora da atividade clínica associada ao aumento da eritropoietina endógena, e a redução dos niveis séricos de ferritina. Isso pode significar mobilização de ferro, assim como, aumento do índice de saturação de transferrina. Estes dados demonstram a importância do Infliximabe na neutralização do efeito inibidor do TNF na produção de eritropoietina. Neste estudo, metade dos pacientes que receberam reposição de ferro venoso apresentou recaída em dez meses, justificando a manutenção do tratamento enquanto houver atividade de doença. 


\section{Deficiências de Vitamina B12 e Ácido Fólico}

As deficiências de Vitamina B12 (cobalamina) e de Vitamina B9 (ácido fólico), embora ocorram em certos pacientes, não apresentam a mesma relevância e prevalência que a deficiência de ferro. No entanto, é importante ficar atento a essas causas adjuvantes de anemia, já que ambas vitaminas do complexo B são absorvidas no íleo. A dosagem dessas vitaminas, no soro, ainda é o exame mais sensível para o seu diagnóstico.

A reposição deve respeitar um princípio que rege o tratamento das doenças crônicas, que é considerar a adesão do paciente ao tratamento. Diante disso, recomenda-se o uso da via oral ou sublingual. A maioria dos autores concorda que a dose diária de 1.000-2.000 mcg de Vitamina B12 Vo ou 500 mcg por via sublingual por um mês pode resolver a anemia na maioria dos pacientes com deficiência de cobalamina, e a dose de $1.000 \mathrm{mcg}$ (VO) ou $500 \mathrm{mcg}$ (SL) mensal como profilaxia ${ }^{30}$. Pacientes com ressecções amplas de íleo ou gastrectomias devem ser suplementados com vitamina B12 parenteral de forma indefinida.

Quanto ao ácido fólico, o uso diário de 5 mg diários é suficiente para tratamento e profilaxia de sua deficiência, assim como durante o uso de medicamentos como a sulfassalazina e o metotrexato.

\section{Prevenção de Anemia na DII}

Medidas preventivas podem ser realizadas visando a detecção precoce de déficits, a correção da dieta, o controle da atividade de doença, suplementações especificas e detecção precoce de mielotoxicidade.

Em pacientes em remissão clínica, o rastreamento de deficiência de ferro deve ser realizado pelo menos anualmente e, se com atividade de doença, pelo menos a cada 3 meses ou intervalos mais curtos, dependendo dos níveis do ferro ${ }^{31 .}$

A determinação anual de ferritina sérica e de vitamina B12 se houver comprometimento ileal deve ser realizada independentemente da presença de atividade inflamatória, tempo de remissão ou do tratamento especifico para DII.

O controle adequado de vitaminas e oligoelementos se faz necessário para uma correta eritropoiese, devendo-se evitar dietas 
de exclusão desnecessárias e suplementação vitamínica se necessária. Na presença de desnutrição associada a hiporexia deve-se avaliar a introdução de nutrição enteral além do controle da atividade de doença.

Pacientes em uso de tiopurinas ou Metotrexato devem ter controles periódicos de hemograma, independente do tempo de tratamento, visando a detecção precoce de mielotoxicidade.

\section{Referências Bibliograficas}

1. Gasche C, Lomer MC, Cavill I, Weiss G. Iron, anaemia, and inflammatory bowel diseases. Gut. 2004. 53:1190-1197.

2. Wells CW, Lewis S, Barton JR, Corbett S. Effects of changes in hemoglobin level on quality of life and cognitive function in inflammatory bowel disease patients. Inflamm Bowel Dis. 2006. 12:123-130.

3. Filmann N ${ }^{1}$, Rey J, Schneeweiss S, Ardizzone S, Bager P, Bergamaschi G, Koutroubakis I, Lindgren S, Morena Fde L, Moum B,Vavricka SR, Schröder O, Herrmann E, Blumenstein I. Prevalence of anemia in inflammatory bowel diseases in european countries: a systematic review and individual patient data meta-analysis. Inflamm Bowel Dis. 2014 . 20(5):936-45.

4. Gomollón F and Gisbert JP. Anemia and inflammatory bowel diseases World J Gastroenterol. 2009; 15(37): 4659-4665.

5. Gasche C. Anemia in IBD: the overlooked villain. Inflamm Bowel Dis 2000. 6:142-50

6. Goodnough L T . Iron deficiency syndromes and iron-restricted erythropoiesis Transfusion 2012. 52 (7): 1584-1592.

7. Gisbert JP, Gomollon F. Common misconcepcions in the diagnosis and management of anemia in inflammatory bowel disease. Am J Gastroenterol 2008;103(5):1299-307

8. Bartels U, Pedersen NS, Jarnum S. Iron absorption and serum ferritin in crohnic inflammatory bowel disease. Scand J Gastroenterol 1978; 13(5):1299-307.

9. Lomer M.C, Kodjabashia K.,. Hutchinson C,. Greenfield S.M, Thompson R.P.,. Powell J.J , Intake of dietary iron is low in patients with Crohn's disease: a case-control study Br J Nutr, 200;. 91 (1) : 141-148

10. Goodnough L Nemeth E, Ganz T, Detection , evaluation and management of iron-restricted erythropoiesis, Blood 2010; 116:4754-61.

11. Munoz M., Garcia-Erce J.A.,. Remacha A.F Disorders of iron metabolism. Part 1: molecular basis of iron homoeostasis J Clin Pathol, 2011; 64 (4) : 281-286

12. Nemeth E, Tuttle MS, Powelson J, Vaughn MB, Donovan A, Ward DM, Ganz T, Kaplan J. Hepcidin regulates cellular iron efflux by binding to ferroportin and inducing its internalization. Science. 2004 ;306(5704):2090-3

13. Crichton RR, Danielsson BG, Geisser P. Iron metabolism: biologic and molecular aspects. In: Crichton RR, Danielsson BG, Geisser P, editors. Iron therapy with special emphasis on intravenous administration. 4th ed. Bremen: UNI-Med Verlag AG, 2008: 14-24

14. Kulnigg S, Gasche C. Systematic review: managing anaemia in Crohn's disease .Aliment Pharmacol Ther 2006; 24 (11-12): 1507-1523

15. Stein, F. Hartmann, A.U. Dignass Diagnosis and management of iron deficiency anemia in patients with IBD Nat Rev Gastroenterol Hepatol, 2011; 7 (11): 599-610

16. Weiss G., Goodnough L.T.. Anemia of chronic disease N Engl J Med, 2005;. 352 (10) : 1011-1023

17. Gasche C.,. Berstad A, Befrits R.,. Beglinger C,. Dignass A,. Erichsen, K et al. Guidelines on the diagnosis and management of iron deficiency and anemia in inflammatory bowel diseases. Inflamm Bowel Dis 2007.; 13 (12): 1545-1553

18. Gasche C.,. Evstatiev R,. Haas T, Kaser A.,. Knoflach P,. Petritsch W, et al.Diagnosis and treatment of iron deficiency and anaemia in inflammatory bowel diseases. Consensus of the Austrian IBD Working Party Z Gastroenterol 2011; 49 (5): 627-632

19. Crichton R. Iron Metabolism, $3^{\text {rd }}$ Edition. Chichester: John Wiley ans Sons Ltd; 2009

20. Ramakrishman U, Yip R, Experiences and challenges in industrialized countries: control of iron 
deficiency in industrialized countries. J Nutr 2002;132:820S-824S

21. Wood RJ, Ronnenberg AG. Iron In: Shils ME, Shike M, Ross AC, Cousins R. Modern Nutrition in health and disease 13th ed. Baltimore: Lippincott Williams \& Wilkins; 2006: 249-270.

22. Pacheco M. Tabela de equivalentes, medidas caseiras e composição química dos alimentos. Aspectos teóricos da nutrição: Minerais. Ed Rubio: 2006: 91-93; RJ,

23. Rubio.Franco G. Tabela de composição química dos alimentos. 9ed. Ed Atheneu: 1997; RJ.

24. Gasche C,Berstad A, Befrits R. Guidelines on the diagnosis and management of iron deficiency and anemia in inflammatory bowel diseases, 2007.13:1545-53

25. Gasche C. Anemia in Inflammatory Bowel disease. Auflage-Brenen:UNI-MED,, 2008

26. Gasche C, Male C, Mayer A et al. Prediction of response to iron sucrose in inflammatory bowel disease associated anemia. Am J Gastroenterol 2001;96(8):2382-7

27. Reinisch W, Staun M, Bhandari S, et al. State of the iron: how to diagnose and efficiently treat iron deficiency anemia in inflammatory bowel disease? J Crohns Colitis. 2013;7:429-40.

28. Gasché C, Dejaco C et al. Intravenous iron and erythropoietin for anemia associated with Crohn disease. A randomized, controlled trial. Ann Intern Med. 1997;126(10):782-7

29. Bergamaschi G, Di Sabatino A et al. Prevalence and pathogenesis of anemia in inflammatory bowel disease. Influence of anti-tumor necrosis factor-alpha treatment. Haematologica. 2010;95(1):199205

30. Nilsson M, Norberg B, Hultdin J, Sandström H, Westman G, Lökk J. Medical Intelligence in Sweden. Vitamin B12: oral compared with Parenteral? Postgraduate Medical Journal 2005;81:191-193.

31. J.D. Cook Diagnosis and management of iron-deficiency anaemia. Best Pract Res Clin Haematol, 2005; 18: 319-332 